\title{
Nanoparticle Temperature Measurements for MEMS Heater Calibration
}

Matthew Mecklenburg' ${ }^{1}$, B. C. Regan ${ }^{2}$ and William Hubbard ${ }^{3}$

${ }^{1}$ University of Southern California, Los Angeles, California, United States, ${ }^{2}$ UCLA Department of Physics \& Astronomy, Los Angeles, California, United States, ${ }^{3}$ NanoElectronic Imaging, Inc., Los Angeles, California, United States

A variety of TEM techniques have demonstrated the ability to detect changes in temperature, but only a few use a sub-10 nm sized focused electron probe. Electron energy loss spectroscopy (EELS) can be used to detect temperature changes through subtle plasmon [1] or phonon [2,3,4] changes. (Many other techniques are being developed and several that examine thermal diffuse scattering [5,6] are also compatible with a small probe.) Here we use Plasmon Energy Expansion Thermometry (PEET) to measure the temperature of drop-cast aluminum nanoparticles located near a MEMS heater. These fiducials are used as nanothermometers to determine the local temperature as a function of the heater power. This approach allows facile in situ thermometric calibration, or calibration verification, of non-commercial and commercial MEMS heaters.

The MEMS heating element appears as a serpentine dark line in the BF STEM image in Figure 1. The Pt wire (thickness: $25 \mathrm{~nm}$ with $5 \mathrm{~nm}$ Ti adhesion layer) and amorphous carbon electrode (thickness: $30 \mathrm{~nm}$ ) were patterned on a silicon nitride window using e-beam lithography. The red ROI in Figure 1 indicates the region near the tip of the carbon electrode where the PEET measurement took place and where we expect the carbon to be hottest with the heater energized. A typical EELS spectrum from one of the aluminum nanoparticles is shown in Figure 1 along with an ADF STEM image of the nanoparticles on the electrode. Spectrum images were acquired at a variety of heater powers. Spectra at each point (example spectrum in Figure 1) were analyzed by fitting both the ZLP and the plasmon peak. The plasmon energy was determined by the energy difference, and the shift in the plasmon energy was converted into a temperature map using the known thermal expansion coefficient of aluminum. The temperature maps at various currents and voltages are shown in Figure 2.

The wire was driven with a maximum current of just over $100 \mu \mathrm{A}$, and reached a maximum power of 132 $\mu \mathrm{W}$ (Figure 2). After some initial drift the heater maintained consistent current-voltage characteristics over tens of minutes. Despite the non-linearity of the current-voltage relation, an analytical expression relating these two quantities can be found using a combination thermal transport and electrical transport equations. The temperatures measured at a few points can then be used to interpolate the temperature near the carbon probe tip at any current, voltage, or power. The temperature shift versus power relation for this sample is linear, with a slope of $3.00 \pm 0.02 \mathrm{~K} / \mu \mathrm{W}$ (Figure 2). Once this relationship is known, temperatures near the electrode for any heater power can be determined.

By measuring the temperature of nanoparticle fiducials with PEET, we can calibrate the heater's temperature vs. power relationship in situ, and a higher degree of reliability can be achieved than with ex situ temperature calibrations. Using nanoparticles or other in situ nanothermometers provides ready access to the local temperature, which allows facile calibration checks before and after an experiment [7]. 

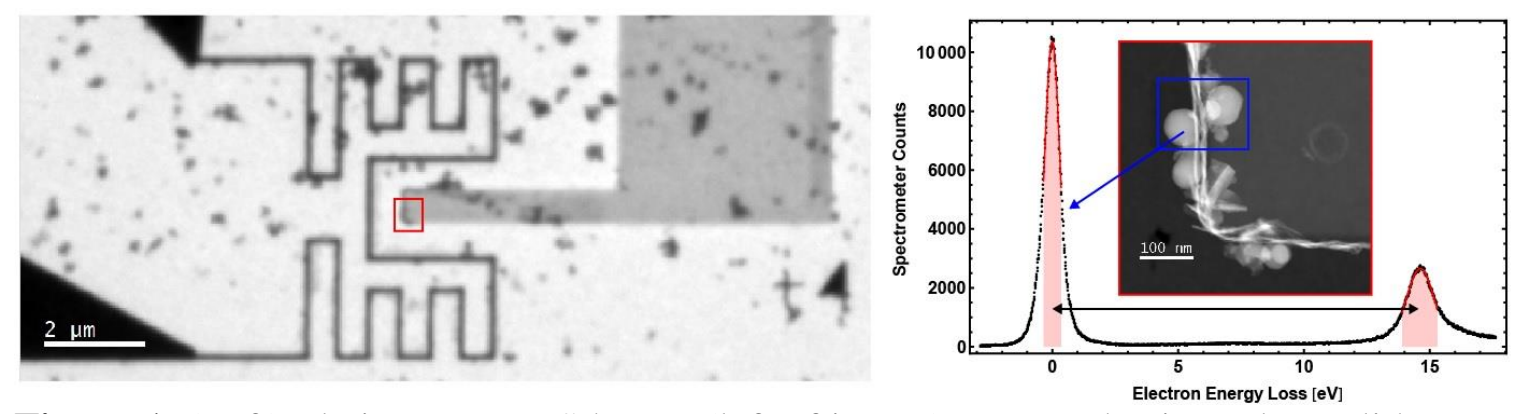

Figure 1. (Left) Platinum MEMS heater (left of image) patterned using e-beam lithography surrounding a carbon electrode (right of image). The black specks are clusters of aluminum nanoparticles. (Right) A spectrum from the center of an aluminum nanoparticle, the highlighted regions of the spectrum showing the portions fit to Gaussian functions, and an ADF STEM image of the aluminum nanoparticles used in this experiment. Individual spectra give temperature measurements with a standard deviation of $14 \mathrm{~K}$.
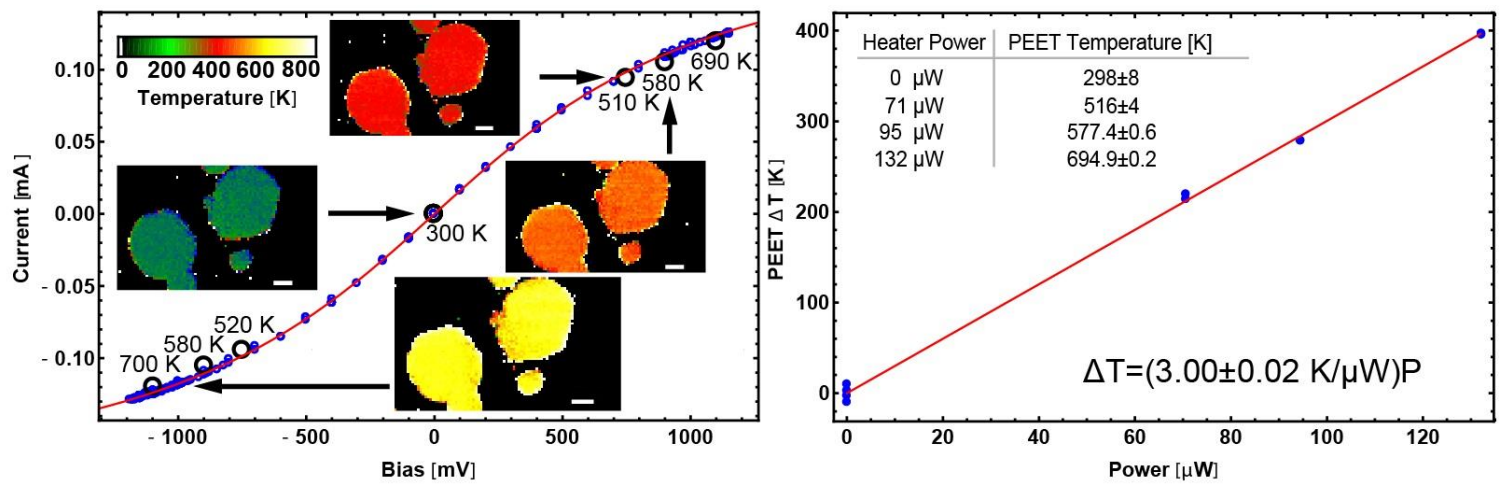

Figure 2. (Left) The current-voltage (IV) relation of the platinum heater and the temperature maps of the aluminum nanoparticles at various points along the IV. The scale bar in each nanoparticle temperature map is $20 \mathrm{~nm}$ wide. (Right) The PEET-determined temperature shift as a function of heater power. The fit relation between power and temperature is shown in the lower right portion of the plot. In the upper left portion is a table comparing the temperature at different powers (both at positive and negative bias, which produces the mean and standard deviation in the inset table).

\section{References}

[1] M Mecklenburg et al., Science 347 (2015), p. 629-632.

[2] X Yan et al., Microscopy and Microanalysis 25 (S2) (2019), p. 622-623.

[3] M Lagos et al., Nano Letters 18 (2018), p. 4556-4563.

[4] J C Idrobo et al., Physical Review Letters 120 (2018), p. 095901.

[5] M Zhu et al., Microscopy and Microanalysis 25 (S2) (2019), p. 1642-1643.

[6] G Wehmeyer et al., Applied Physics Letters 113 (2018), p. 253101.

[7] This material is based upon work supported by the Defense Microelectronics Activity under Contract No. HQ072720P0004. 